Review

\title{
Mind the gap: connexins and cell-cell communication in the diabetic kidney
}

Claire E. Hills, Gareth W. Price, Paul E. Squires

School of Life Sciences, University of Lincoln, Brayford Pool, Lincoln LN6 7TS, UK

Address for correspondence:

Claire E. Hills, Email: chills@lincoln.ac.uk

\begin{abstract}
Connexins, assembled as a hexameric connexon, form a transmembrane hemichannel that provides a conduit for paracrine signalling of small molecules and ions to regulate the activity and function of adjacent cells. When hemichannels align and associate with similar channels on opposing cells, they form a continuous aqueous pore or gap junction, allowing the direct transmission of metabolic and electrical signals between coupled cells. Regulation of gap junction synthesis and channel activity is critical for cell function, and a number of diseases can be attributed to changes in the expression/function of these important proteins. Diabetic nephropathy is associated with several complex metabolic and inflammatory responses characterised by defects at the molecular, cellular and tissue level. In both type 1 and type 2 diabetes, glycaemic injury of the kidney is the leading cause of end-stage renal failure, a consequence of multiple aetiologies, including increased deposition of extracellular matrix,
\end{abstract}


glomerular hyperfiltration, albuminuria and tubulointerstitial fibrosis. In diabetic nephropathy, loss of connexin mediated cell-cell communication within the nephron may represent an early sign of disease; however, our current knowledge of the role of connexins in the diabetic kidney is sparse. This review highlights recent evidence demonstrating that maintained connexin-mediated cell-cell communication could benefit region-specific renal function in diabetic nephropathy and suggests that these proteins should be viewed as a tantalising novel target for therapeutic intervention.

Keywords Cell-cell communication, Connexins, Diabetic nephropathy, Gap junction, Hemichannel, Review

Received: 2 May 2014 / Accepted: 25 September 2014 


$\begin{array}{ll}\text { Abbreviations } \\ \alpha-S m a & \text { alpha Smooth muscle actin } \\ \text { Cx } & \text { Connexin } \\ \text { E-cadherin } & \text { Epithelial cadherin } \\ \text { EMT } & \text { Epithelial-to-mesenchymal transition } \\ \text { ESRD } & \text { End-stage renal disease } \\ \text { GJIC } & \text { Gap junction intercellular communication } \\ \text { NCAD } & \text { Neural cadherin } \\ \text { STZ } & \text { Streptozotocin } \\ \text { ZDF } & \text { Zucker Diabetic Fatty } \\ \text { ZL } & \text { Zucker Lean } \\ \text { ZO-1 } & \text { Zonula occludens 1 }\end{array}$

\section{Introduction}

Efficient renal function depends on cooperation between cells of the nephron and cells of the renal vasculature. Gap junctions permit direct intercellular transfer of small molecules and ions and are formed from the alignment of two connexons on adjoining cells [1]. Each connexon is composed of a hexameric assembly of connexins that make up a transmembrane pore called a hemichannel [1]. When hemichannels align with those on neighbouring cells they 'dock' to form an aqueous pore permitting the transmission of metabolic and electrical signals between coupled cells that facilitates cellular activity and synchronises tissue function. Most cells communicate with their immediate neighbours via gap junctions, and multiple connexin isoforms are expressed in the kidney [2]. In the kidney, multiple connexin isoforms are expressed [2]. However, whilst the presence of gap junctions has long been known, we have only recently begun to understand how connexins regulate renal function. Expressed in the renal vasculature, connexins have been demonstrated to contribute to the regulation of both GFR and renin secretion, thus suggesting a role for connexins in the regulation of blood pressure [3-4]. This critical function rightly warrants our 
attention. However, connexins are also highly expressed in tubular epithelium, where their role is far less certain. Elevated glucose in diabetes dramatically alters connexin expression, and it seems likely that changes in cell-cell communication play a critical role in the aetiology and pathophysiology of the disease [5-10].

In patients with diabetes, nephropathy represents the leading cause of end-stage renal disease (ESRD) and is the leading cause of entry into the renal replacement therapy programme. Sadly, renal complications of diabetes account for approximately $21 \%$ of deaths in patients with type 1 and approximately $11 \%$ of deaths in patients with type 2 [11]. As the incidence of diabetes continues to increase, there is a pressing demand to identify future therapeutic targets to alleviate debilitating complications such as diabetic nephropathy. Associated with complex metabolic and inflammatory changes, diabetic nephropathy is characterised by defects at the molecular, cellular and tissue level [12]. Alterations in the expression of key candidate proteins pivotal to cell communication could contribute to the development and progression of key underlying pathologies of diabetic nephropathy. In the current article we review expression, localisation and function of connexin throughout the diabetic kidney and explore how glucose and its downstream pro-fibrotic mediators alter these important proteins.

\section{Connexins, connexons, gap junctions and hemichannels}

Connexins are a family of transmembrane proteins critical for intercellular communication. In humans, there are 21 genes known to be associated with the connexin family. Commonly designated by numerical suffixes referring to their molecular weight, the expression of these proteins may be ubiquitous (Cx32, Cx37, Cx40, Cx43 and Cx45) or tissue specific, e.g. Cx32 is expressed in numerous cells, including cardiac tissue, the kidney and hepatocytes, whilst Cx62 is expressed only in retinal tissue [13]). The biosynthesis and export of connexin polypeptides to the cell membrane is similar to other membrane bound 
proteins, with membrane insertion denoting topographic arrangement [14]. However, with a half-life of only a few hours, connexins are subjected to a continuous cycle of synthesis and degradation, a process that allows for acute regulation of channel activity and gap junction-mediated cell communication [14].

Sharing common structural features, connexins are composed of four transmembrane helices interconnected by two extracellular loops, one intracellular loop, and cytoplasmic $-\mathrm{NH}_{2}$ and $-\mathrm{COOH}$ terminal regions. Whilst the highly conserved $-\mathrm{NH}_{2}$ terminal tail incorporates a putative calmodulin-binding motif, which is necessary for membrane insertion, both the $-\mathrm{NH}_{2}$ terminal tail and $-\mathrm{COOH}$ terminal region are thought to play a central role in the regulation of channel conductance, being controlled by phosphorylation and post-translational modification [15, 16]. Phosphorylation of connexins can occur on either serine/threonine or tyrosine residues, and changes in conductance and/or permeability may have implications for the assembly, degradation and biosynthesis of gap junctions. [17]

Gap junctions are formed through interaction between two connexons, or hemichannels on the membrane of adjacent cells. Hexameric structures comprised of six individual connexins, the resultant junction contains a 1-2 nm diameter aqueous pore, through which ions and small molecules, including $\mathrm{Ca}^{2+}$, ATP, cAMP, $\mathrm{Na}^{+}$and $\mathrm{IP}_{3}$ pass [18]. Depending on the combination of connexin isoforms, they can either be homomeric or heteromeric (See Fig 1). As standalone structures, hemichannels form when connexons are inserted into the membrane without association with another connexon on a neighbouring cell [18]. Hemichannels are also permeable to a variety of small metabolites and have been shown to contribute to paracrine signalling [19]

The composite connexin isoforms of a given gap junction determine the selectivity of molecules able to migrate through the junction (i.e. its permeability), which, in turn, is determined by the diameter and charge of the pore formed. For 
example, Cx43 exhibits a greater affinity for AMP, ADP and ATP as compared with Cx32, whilst Cx32 has a 12-fold higher affinity for adenosine [20]. It is the ability of different isoforms to combine that lends itself to diversity in terms of permeability, selectivity and conductance, with conductance not just controlled by isoform homology but also by various stimuli, including $\mathrm{pH}, \mathrm{Ca}^{2+}$ and posttranslational modification. It remains to be determined whether gap junction assembly and membrane insertion is random or site directed. What is known is that extracellular matrix (ECM) proteins, cadherins, integrins and laminins are required to facilitate their adhesion once inserted into the membrane [21-22].

The number of diseases attributed to mutations and single nucleotide polymorphisms in proteins involved in regulation of gap junction synthesis and channel activity suggest that functional gap junctions are critical for cell function [23]. Connexins have a role in ischaemia [24], inflammation [25] and both renininduced and essential hypertension [26-27]. Furthermore, the relationship between hyperglycaemia and a loss of gap junction expression is important in the progression and development of multiple microvascular complications in diabetes [28]. Effects of hyperglycaemia on gap junction intercellular communication (GJIC) have been examined in multiple tissue types, and there are reports that glucose decreases gap junction conductance and disrupts cellular homeostasis in various cell systems, including retinal pericytes [5], endothelial cells [6] and epithelial cells [29]. A glucose-evoked reduction of $\mathrm{Cx} 43$ in retinal capillaries of diabetic mice is observed to result in, increased apoptosis, loss of cell communication and a decline in pericyte and acellular capillary number [5]. Loss of $\mathrm{Cx} 43$ in vascular cells of diabetic rats impairs ventricular induction, suggesting that a loss of GJIC may also contribute towards cardiovascular complications of diabetes [30]. However instigated, it is clear that a glucose-induced loss of connexin-mediated cell-cell communication is important in the development of several facets of this debilitating metabolic disease. 


\section{The role of connexins in the diabetic kidney}

In the kidney, multiple structural and functional changes occur in response to elevated levels of glucose, including those that occur in the glomerulus, tubulointerstitium and vasculature [12]. Structural abnormalities range from thickening of the glomerular basement membrane, hypertrophy, interstitial fibrosis and tubular atrophy [12]. Together, these changes contribute to glomerular hyperfiltration, albuminuria, systemic hypertension and loss of renal function [12]. Of the 21 connexin isoforms expressed in humans, nine are found in the tubular and vascular regions of the kidney (Cx26, Cx31, Cx30.3, Cx37, Cx32, Cx40, Cx43, Cx45 and Cx46) (for a review, see [2]). However, our understanding of how changes in gap junctions in the kidney facilitate progression of diabetes is limited. The following sections discuss our current knowledge for a role of connexins in the nephron, specifically, the renal vasculature, mesangium and podocytes.

Vasculature Within endothelial cells of the rat afferent arteriole, Cx37, Cx40 and Cx43 are present, whilst post-glomerular vasculature appears to express only Cx43 [2]. For cells of the pre-glomerular vessels to communicate efficiently, they must be functionally coupled. Loss of synchronised cell communication may predispose to the development of early changes that underline the onset of diabetic nephropathy. This notion is supported by the presence of modified levels of both Cx40 and Cx43 in streptozotocin (STZ)-treated mice. In non-diabetic mice, Cx40 is expressed on the endothelium from afferent arterioles, whilst $\mathrm{Cx} 43$ is localised to efferent arterioles. However, following STZ ablation of insulin-secreting pancreatic beta cells, expression of $\mathrm{Cx} 40$ was found to be increased in afferent arterioles and inside the glomerulus, whilst expression of endothelial Cx43 in efferent arterioles was decreased [7-8].

The role of $\mathrm{Cx} 40$ in regulating blood pressure by modulating renin secretion is well documented. Renin is released by the juxtaglomerular apparatus and 
controls extracellular volume through changes in angiotensin II and mean arterial pressure [31]. Renin-producing cells are linked by $\mathrm{C} x 40-$ mediated gap junctions and are highly electrically coupled [32-33]. Knocking out the gene encoding Cx40 (Gja5 ${ }^{1-}$ mice) makes these animals hypertensive [32].

It has previously been reported that renin-dependent hypertension experimentally induced by the clipping of a renal artery in the two-kidney, one-clip (2K1C) model is associated with a vessel-specific increase in the expression of Cx43 [34]. However, when $\mathrm{C} \times 43$ expression is repressed and replaced with $\mathrm{C} \times 32$, there is a marked reduction in renin secretion and mice are normotensive [35]. These findings have recently been questioned by Gerl $M$ et al who, using mice with endothelium and renin cell-specific deletions of $\mathrm{Cx} 43$, failed to confirm a requirement for $\mathrm{C} \times 43$ in either renin expression or secretion in animals on either a normal salt diet or during chronic challenge of the renin system by pretreatment with a low-salt diet in the absence or presence of an ACE inhibitor [36].

In addition to controlling renin secretion, there is a link between connexins and glomerular hyperfiltration. Takenaka et al examined altered gap junction communication and abnormalities in renal haemodynamic in the Zucker Lean (ZL) rat and the Zucker Diabetic Fatty (ZDF) rat model of type 2 diabetes [37]. They reported that ZDF rats exhibit glomerular hyperfiltration with impaired autoregulation. The increase in GFR was linked to increased phosphorylation of Cx43 and reduced $\mathrm{C} \times 37$ expression in renin-secreting cells, suggesting that impaired GJIC in the juxtaglomerular apparatus mediates, or in part contributes to, altered renal autoregulation in the disease [37]. These data suggest that dysregulation of $\mathrm{C} \times 37, \mathrm{C} \times 40$ and $\mathrm{C} \times 43$ contribute to impaired autoregulation, glomerular hyperfiltration and dysregulated blood pressure homeostasis.

Mesangium Ultrastructural and histochemical studies indicate the existence of a high density of gap junctions among mesangial cells, but little is known about their regulation. In diabetic nephropathy, mesangial cell proliferation has been 
proposed to play a role in the progression of glomerulosclerosis [38-39]. Glomerular mesangial cells are highly coupled by $\mathrm{Cx} 43$-containing gap junctions, with expression levels mirrored by GJIC [40]. Expression levels of Cx43 are downregulated in mesangial and endothelial cells of STZ-induced diabetic rats [41], whilst exposure of rat glomerular mesangium to platelet-derived growth factor evokes a phosphoinositide 3-kinase-dependent loss of Cx43-mediated GJIC [42]. This loss of cell communication, Yao et al propose, may in part contribute to the complex interplay of events that accompany mesangial cell proliferation in forms of renal disease, including diabetic nephropathy [42]. In support of these studies, recent findings by Morioka et al have confirmed that Cx40 expression in mesangial cells is related to mesangial cell regeneration, suggesting that connexin expression could be a therapeutic target for glomerular disease [43].

Mesangial expansion is a hallmark of early diabetic nephropathy and is regarded as the structural variable that best correlates with dysregulated GFR, proteinuria and hypertension [44]. Exposure of cultured glomerular mesangial cells to high glucose evokes arrest in the G1 (senescence) phase of the cell cycle [45]. Zhang et al confirmed that a glucose-evoked reduction in $\mathrm{Cx} 43$ accelerated the progression of mesangial cells through senescence, a state of arrested cell growth linked to mesangial cell hypertrophy in diabetic nephropathy [45]. In support of this, glucose-induced hypertrophy of mesangial cells was reversed by overexpressing Cx43 via the phosphatase and tensin homologue/Akt/mechanistic target of rapamycin (PTEN/Akt/mTOR) pathway [46]. These studies suggest that high glucose levels downregulate mesangial Cx43 expression, inducing cell cycle arrest, inhibition of proliferation and promotion of protein synthesis and hypertrophy of the mesangium, thus highlighting the possibility of $\mathrm{Cx} 43$ as a future therapeutic target to both modulate cell hypertrophy and prevent mesangial expansion in diabetic nephropathy [46].

Through interactions with numerous cell signalling and cell scaffold proteins, connexins are known to play a crucial role in cell adhesion, proliferation and 
migration. The carboxy tail of $\mathrm{Cx} 43$ interacts with c-Src, a non-receptor tyrosine kinase that can regulate cell proliferation. Activated c-Src phosphorylates Cx43 on tyrosine residues Tyr265 and Tyr247, ultimately impairing and reducing GJIC [47-48]. Elevated levels of c-Src have been linked to the pathogenesis of diabetic nephropathy [49]. Furthermore, studies by Xie et al revealed that overexpression of Cx43 or inhibition of C-Src not only attenuates the upregulation of glucoseinduced intercellular adhesion molecule 1 (ICAM1), TGF- $\beta 1$ and fibronectin expression in glomerular mesangial cells, but promotes and contributes to nuclear factor-KB activation, leading to renal inflammation [41].

Abnormalities in renal NO generation have also been linked to the pathogenesis of renal disease in diabetes [50-52]. Whilst intrarenal NO production mediated primarily by constitutively released $\mathrm{NO}$ is increased in the early phases of diabetic nephropathy, a progressive decline in NO production and, specifically, NO bioavailability in the kidney has been observed with advancing renal failure [53]. A potential role of $\mathrm{NO}$ in the regulation of gap junction intercellular communication was suggested by Yao et al, who confirmed that increased NO augments Cx43-mediated GJIC; an effect they found to be dependent on protein kinase A [54]. Their observations were the first to suggest that diminished NO in diabetic nephropathy may in part mediate a loss of gap junction-mediated cell communication in mesangial cells.

In addition to contributing to gap junction formation, uncoupled hemichannels permit local paracrine release of nucleotides (See Fig. 2) [19]. The gating of hemichannels is controlled by various physiological stimuli, including changes in membrane depolarisation, ionic concentration, mechanical stimulation and metabolic inhibition.

Like gap junctions, hemichannels have a role in both cell survival and cell death [19]. Hemichannel-mediated ATP release has recently been linked to the progression and development of fibrosis in cardiac fibroblasts, data that suggests a potential pathophysiological role for dysregulated hemichannel-mediated ATP 
signalling [55]. Oxidative stress can open Cx43 hemichannels [56]. A recent study examined a role for boldine, an alkaloid with antioxidant, antiinflammatory, and hypoglycaemic effects in the reversal of renal complications in the STZ-induced diabetic rat [57]. The study demonstrated that under resting conditions, mesangial hemi-channels had a low open-state probability. However, glucose, IL-1 $\beta$ and TNF- $\alpha$ all increased hemichannel activity, even at negative membrane potential and in the presence of extracellular divalent cations [57]. Furthermore, when exposed to high glucose and proinflammatory cytokines, mesangial cells exhibited reduced gap junction-mediated cell coupling, effects that were reversed by co-administration with boldine. Interestingly, cells cultured in high glucose in the presence of proinflammatory cytokines, exhibited a slight, but insignificant increase in Cx43 expression, whilst addition of boldine to the high glucose and proinflammatory cytokines led to a statistically significant elevation in Cx43 expression as compared with control. These results demonstrate that when exposed to a diabetic milieu, mesangial cells exhibit increased hemichannel activity and impaired gap junction-mediated cell communication-an effect abolished by co-incubation with boldine [57]. The authors suggest that the boldine-induced increase in Cx43 may account for newly synthesised gap junctions and explain the increase in coupled cells when co-administered with proinflammatory cytokines. Boldine may protect the kidney against oxidative stress by preventing hemichannel opening and ensuring cells remain successfully coupled to each other via gap junctions.

Podocytes Impaired renal function in diabetic nephropathy is a consequence of a number of underlying pathologies that ultimately stem from the loss in viable nephrons. In the majority of cases, this process is initiated in response to podocyte injury. Whilst podocyte injury or podocyte loss in the renal glomerulus has been proposed as the crucial mechanism in the development of glomerulosclerosis, our understanding of how podocytes respond to injury is limited. Previous studies have shown that $\mathrm{Cx} 43$ is expressed on podocytes in 
both normal and diseased kidney tissue [58-59]. Initial studies by Yaoita et al reported increased Cx43 expression levels in the early to nephrotic stage of puromycin aminonucleoside-induced nephrosis in rats, an event they suggest may represent an early marker of podocyte injury [60]. Further to these findings, and given that oxidative stress mediates podocyte injury under a variety of pathological situations, Yan et al have reported that exposure of podocytes to puromycin evokes an increase in both NADPH oxidase 4 (NOX4) expression and superoxide generation [61]. Furthermore, inhibition of NADPH oxidase attenuated the puromycin-induced increase in $\mathrm{C} x 43$ expression, whilst treatment of podocytes with several structurally different gap junction inhibitors significantly attenuated the cytotoxicity of puromycin, thus suggesting that NADPH oxidasemediated upregulation of $\mathrm{Cx} 43$ contributes to podocyte injury [61]. Finally, failure of Lucifer yellow dye to transfer between parietal epithelial cells of the Bowman's capsule, led to the suggestion that podocytes may respond to injury as an integrated epithelium on a glomerulus rather than individually as a separate cell [60].

Our knowledge of podocyte gap junctions and their role in diabetic kidney disease is sparse. Studying renal biopsies and podocytes from patients with type 2 diabetes and comparing these with nephrectomised kidney samples from individuals with localised neoplasm or minor glomerular abnormalities, Sawai et al [62] observed a loss of $\mathrm{Cx} 43$ expression in patients with overt nephropathy. To retain renal function, the loss of podocytes and glomerular hypertrophy in diabetic nephropathy must be compensated for. To this end, podocytes migrate and extend their cell body over the glomerular basement membrane depleted of functioning podocytes. Sawai et al proposed that a loss of Cx43 and redistribution away from points of cell-cell contact, impaired podocyte cell communication and contributed to the loss of barrier function leading to albuminuria [62]. They hypothesised that $\mathrm{C} \times 43$ directly interferes with tight junctions and cytoskeletal proteins, ultimately impairing slit diaphragm components essential for the selective filtering process. As a consequence of this important study, Cx43 staining may represent an easy and convenient means of 
assessing podocyte damage and renal function in diabetic nephropathy.

\section{Connexins, cell-cell communication and renal fibrosis}

Diabetic nephropathy is histologically characterised by increased expression of interstitial matrix components and an accumulation of the ECM in both the glomerular mesangium and tubular interstitum, together culminating in excessive renal scarring and a decline in excretory function [12]. Renal fibrosis presents as glomeruosclerosis, tubulointerstitial fibrosis, infiltration of inflammatory mediators and the activation of alpha-smooth muscle actin-positive myofibroblasts [63-64]. Of these, tubulointerstitial fibrosis is the key pathology underlying diabetic nephropathy, with fibrosis and the subsequent loss of cell function being a predictive marker for ESRD. Understanding the signals that regulate the deposition of fibrotic material in the interstitium is important for developing sitedirected therapies that may help alleviate this damage.

Central to tubulointerstitial fibrosis is tubular epithelial-to-mesenchymal transition (EMT), or the trans-differentiation of tubular epithelial cells into myofibroblasts [63]. In diabetic nephropathy, EMT occurs as cells attempt to avoid apoptosis in the presence of high glucose. Ultimately, cells undergo a number of morphological and phenotypic changes in which they lose classic epithelial characteristics in exchange for markers more commonly associated with a mesenchymal phenotype. This trans-differentiation occurs at four distinct stages. The pivotal step of EMT is denoted by a loss of epithelial (E)-cadherin and the subsequent loss of cell-cell adhesion. This is further accompanied by a loss of epithelial proteins central to tight junction formation, e.g. claudin and the zonula occludens protein (ZO-1), whilst cytoskeletal remodelling is accompanied by the loss of cytokeratin expression, replaced by the intermediate filament protein vimentin and alpha-smooth muscle actin. Together, these morphological changes result in loss of cell adhesion and tubular basement membrane disruption which, when combined with expression of fibroblast specific protein 1, results in the 
production of a newly defined group of cells that exhibit enhanced motility and increased proliferative and contractile capacity. These cells migrate from the tubular basement membrane into the interstium where they reside as fibrotic mediators, an event that leads to increased deposition of the ECM [64].

Tissue integrity is maintained by cell-cell and cell-substrate (ECM) adhesion. Adhesion is multifunctional, providing structural support and a link between the cell and its environment. Loss of adhesion precedes loss of function. In diabetic nephropathy, impaired E-cadherin mediated cell-cell adhesion represents a pivotal step in early proximal tubular injury [63-64]. Cadherins help form the multiprotein adherens junction that links cell-cell contact to the actin cytoskeleton and various signalling molecules. In epithelial cells connexins co-localise with cadherins and, consequently, gap junctions form close to the site of intercellular adhesion, where they mediate GJIC [21]. Inhibiting cadherin-based cell adhesion inhibits gap junction assembly and impairs cell-cell communication [65], while upregulation of E-cadherin-dependent cell-cell contacts increased GJIC in mouse epidermal cells [66]. In human proximal tubule cells, glucose-evoked increases in TGF- $\beta 1$ reduced Cx43-mediated GJIC by reducing cell-cell adhesion [67]. Interestingly, some type 2 diabetic patients have been found to have elevated levels of visfatin, and this adipokine has recently been shown to inhibit expression of both Cx26 and Cx43 in the human kidney (HK2) proximal tubule cell line [68].

In wound healing, deposition of the ECM is a response to renal injury, whilst excessive matrix deposition is a hallmark of fibrosis and occurs when cells of the nephron over compensate in their attempt to maintain function [69]. Therefore, a tight balance between synthesis and breakdown of matrix proteins is required. One mechanism of control is through regulation of collagen and matrix metalloproteinase (MMP) production [70]. Loss of this regulation can tip the balance from repair to injury, culminating in a build-up of fibrotic material and scar formation. Recent studies examining the role of connexins in wound healing in human diabetic fibroblasts and keratinocytes suggest that an increase in cell 
motility may occur as a consequence of decreasing both gap junction and hemichannel activity [71]. Furthermore, keratinocytes exhibit a loss of Cx43 expression at 24-48 $\mathrm{h}$ during the normal healing process as cells become migratory in an attempt to close the wound [72]. These studies suggest that a loss of connexin expression may facilitate wound healing, an observation that may have dramatic implications for scar formation and fibrosis in the diabetic kidney. In an STZ-induced mouse model, cell migration is delayed until the connexins are downregulated [73]. Connexins form partnerships with multiple adhesion and structural proteins, e.g. Cx43 forms a complex with ZO-1, $\beta$-catenin and $\mathrm{N}$-cadherin, proteins likely to influence cell adhesion and wound healing. Not surprisingly, knockdown of $\mathrm{Cx} 43$ in conjunction with the cell adhesion protein $\mathrm{N}$ cadherin accelerates cell migration in a scratch wound healing assay [74]. These data, which are consistent with those presented by Hills et al [67] confirming a link between loss of Cx expression and impaired E-cadherin-mediated cell adhesion in the human proximal tubule, support the notion that both Cx43 and adhesion proteins may represent therapeutic targets for improved wound repair and in alleviating tubulointerstital fibrosis, where loss of connexin expression in the renal tubule may not only lead to impaired cell communication but may facilitate increased cell migration. In accordance with morphological and phenotypic changes linked to early tubular injury in diabetic nephropathy, loss of connexin-mediated communication and increased motility may facilitate the migration of cells into the interstitium, where they can reside as newly defined fibrotic mediators.

Contrary to the above, recent studies by Abed et al propose an opposite effect of impaired $\mathrm{Cx}$ expression. Having confirmed that biopsies of patients with chronic kidney disease exhibit elevated levels of $\mathrm{Cx} 43$, they also confirmed that, when interbred with RenTg mice, a genetic model of hypertension-induced chronic kidney disease, $\mathrm{C} \times 43^{+/-}$mice exhibit a marked decrease in cell adhesion markers, which leads to reduced monocyte infiltration and interstitial renal fibrosis. In addition, functional and histological markers such as albuminuria and glomerulosclerosis were also ameliorated [75]. These differences in both 
expression and function however may of course be region specific and may be explained by an absence of hyperglycaemia.

\section{Do connexins protect against diabetic nephropathy?}

In the cortical collecting duct, efficient and appropriate re-uptake of sodium depends upon an integrated response of the ductal epithelium to changes in osmolarity. This in turn relies on connexin-mediated coordination of cellular activity [76-77]. Abnormal sodium reabsorption has been linked to the development of hypertension in both renal disease and diabetic nephropathy. Cells of the human cortical collecting duct express Cx43 and the transient receptor potential cation channel subfamily $V$ member 4 (TRPV4) ion channels [78]. Mechano-sensitive TRPV4 channels regulate cell volume via $\mathrm{Ca}^{2+}$ dependent mechanisms [79] and help maintain epithelial integrity and ductal function in response to osmotic challenge by high glucose. Inability to resolve osmotic changes has serious repercussions for the transport of $\mathrm{Na}^{+}$and the development of secondary hypertension associated with renal diseases, such as diabetic nephropathy. Cx43 mediates GJIC between cells of the collecting duct, and high glucose $(25 \mathrm{mmol} / \mathrm{l})$ actually increases this expression and consequently improves cell-cell communication [80]. These data suggest a pivotal role for Cx43-mediated gap junctions in the synchronisation of activity between cells of the cortical collecting duct in response to stimuli that mimic the osmotic and physical challenges associated with high glucose. To date, there is a wealth of information relating loss of gap junction expression and impaired cell communication to various secondary complications of diabetes in multiple tissue types [28]. In all instances, reduced Cx expression was shown to evoke pathophysiological changes through impaired GJIC, which ultimately facilitated loss of cell communication and impaired function. However, in the cortical collecting duct, it is interesting to speculate that glucose-evoked increases in $\mathrm{Cx}$ expression and enhanced cell communication may protect the duct from further glucose-induced damage, including alterations in re-absorptive capacity, associated with more established, progressive diabetic nephropathy. 
The functional consequences of these data remain to be confirmed, and it should be noted that some cells change their $\mathrm{Cx}$ expression pattern in culture [81]. Investigators should bear this in mind when extrapolating in vitro cell work to the clinical scenario.

\section{Conclusion}

To help synchronise activity and coordinate function within different regions of the nephron, connexins mediate direct gap junction-associated cell-cell communication as well as local paracrine intercellular signalling from hemichannels. In diabetic nephropathy, these small proteins play a vital role in orchestrating an integrated functional response against osmotic, fibrotic and metabolic assault. This review outlines growing evidence that connexins offer a viable future therapeutic target for the control and treatment of a number of renal diseases, including diabetic nephropathy.

\section{Acknowledgements}

This work was supported by the generous support of Diabetes UK (BDA:11/0004215 and BDA:12/0004546) and by an EFSD/Janssen grant.

\section{Contribution statement}

All authors contributed to drafting the manuscript and have approved the final version for publication.

\section{Duality of Interest}

The authors declare that there is no duality of interest associated with this 
manuscript.

\section{References}

1.Bosco D, Haefliger JA, Meda P (2011) Connexins: key mediators of endocrine function. Physiol Rev 91:1393-445

2. Hanner F, Sorensen CM, Holstein-Rathlou NH, Peti-Peterdi J (2010) Connexins and the kidney. Am J Physiol Regul Integr Comp Physiol 298:R1143R1155

3. Oppermann M, Carota I, SchiessI I, Eisner C, Castrop H, Schnermann J (2013) Direct assessment of tubuloglomerular feedback responsiveness in connexin 40deficient mice. Am J Physiol Renal Physiol 304:F1181-F1186

4. Kurtz A (2012) Renal connexins and blood pressure. Biochim Biophys Acta 1818:1903-8

5. Bobbie MW, Roy S, Trudeau K, Munger SJ, Simon AM, Roy S (2001) Reduced connexin 43 expression and its effect on the development of vascular lesions in retinas of diabetic mice. Invest Opthalmol Vis Sci 51:3758-3763

6. Li AF, Roy S (2009) High glucose induced downregulation of connexin 43 expression promotes apoptosis in microvascular endothelial cells. Invest Opthalmol Vis Sci 50:1400-1407

7.Zhang J, Hill CE (2005) Differential connexin expression in preglomerular and postglomerular vasculature: accentuation during diabetes. Kidney Int 68:11711185

8.Zhang JH, Kawashima S, Yokoyama M, Huang P, Hill CE (2006) Increased eNOS accounts for changes in connexin expression in renal arterioles during diabetes. Anat Rec A Discov Mol Cell Evol Biol 288:1000-1008 
9.Li AF, Sato T, Haimovici R, Okamoto T, Roy S (2003) High glucose alters connexin 43 expression and gap junction intercellular communication activity in retinal pericytes. Invest Ophthalmol Vis Sci 44:5376-5382

10.Sato T, Haimovici R, Kao R, Li AF, Roy S (2002) Downregulation of connexin 43 expression by high glucose reduces gap junction activity in microvascularendothelial cells. Diabetes 51:1565-1571

11. http://www.diabetes.org.uk/documents/reports/diabetes_in_the_uk_2010.pdf

12.Wada J, Makino H (2013) Inflammation and the pathogenesis of diabeticnephropathy. Clin Sci 124:139-52

13.Masahito Oyamada Kumiko Takeb, Yumiko Oyamada (2013) Regulation of connexin expression by transcription factors and epigenetic mechanisms. Biochimica et Biophysica Acta (BBA) - Biomembranes. 1828:118-33

14.Laird DW (2006) Life cycle of connexins in health and disease. Biochem J 394:527-543

15.Kronengold J, Srinivas M, Verselis K (2012) The N-terminal half of the connexin protein contains the core elements of the pore and voltage gates. $J$ Membr Biol 245:453-463

16. Herve JC, Bourmeyster N, Sarrouilhe D, Duffy HS (2007) Gap junctional complexes: From partners to functions. Prog. Biophys. Mol Biol 94:29-65

17.Lampe PD, Lau AF (2004) The effects of connexin phosphorylation on gap junctional communication. Int J Biochem Cell Biol 36:1171-86

18.Nielsen MS, Nygaard Axelsen L, Sorgen PL, Verma V, Delmar M, HolsteinRathlouNH (2012) Gap Junctions. Compr Physiol 2:1981-2035

19.Wang N, de Bock M, Decrock E et al (2013) Paracrine signaling through plasmamembrane hemichannels. Biochim Biophys Acta 1828:35-50 
20.Goldberg GS1, Alexander DB, Pellicena P, Zhang ZY, Tsuda H, Miller WT (2003)Src phosphorylates Cas on tyrosine 253 to promote migration of transformed cells. J Biol Chem 278:46533-40

21.Fujimoto K, Nagafuchi A, Tsukita S, Kuraoka A, Ohokuma A, Shibata Y (1997) Dynamics of connexins, E-cadherin and alpha-catenin on cell membranes during gap junction formation. J Cell Sci 110:311-22

22.Defamie N, Chepied A, Mesnil M (2014) Connexins, gap junctions and tissue invasion. FEBS Lett 17;588:1331-1338

23.Pfenniger A, Wohlwend A, Kwak BR (2011) Mutations in connexin genes and disease. Eur J Clin Invest 41:103-16

24.Sahu G, Bera AK (2013) Contribution of intracellular calcium and pH in ischemic uncoupling of cardiac gap junction channels formed of connexins 43 , 40, and 45: a critical function of C-terminal domain. PLoS One 8:e60506

25.Tsuchida S, Arai $Y$, Kishida $T$ et al (2013) Silencing the expression of connexin 43 decreases inflammation and joint destruction in experimental arthritis. J Orthop Res 31:525-30

26. Le Gal L, Alonso F, Wagner C et al (2014) Restoration of connexin 40 (Cx40) in renin-producing cells reduces the hypertension of $\mathrm{Cx} 40$ null mice. Hypertension 63:1198-1204

27. Firouzi M, Kok B, Spiering $W$ et al (2006) Polymorphisms in human connexin40 gene promoter are associated with increased risk of hypertension in men. J Hypertens 24:325-30

28. Wright JA, Richards T, Becker DL (2012) Connexins and diabetes. Cardiol Res Pract 2012:496904

29. Tien T, Barrette KF, Chronopoulos A, Roy S (2013) Effects of high glucoseinduced $\mathrm{C} \times 43$ downregulation on occludin and ZO-1 expression and tight junction 
barrier function in retinal endothelial cells. Invest Ophthalmol Vis Sci 3:54:651825

30. Inoguchi T, Yu HY, Imamura M et al (2001) Altered gap junction activity in cardiovascular tissues of diabetes. Medical Electron Microscopy 34:86-91

31.Wagner C, Kurtz A (2013) Distribution and functional relevance of connexins in renin-producing cells. Pflugers Arch 465:71-7

32. Krattinger N, Capponi A, Mazzolai $L$ et al (2007) Connexin40 regulates renin production and blood pressure. Kidney Int 72:814-22

33.Takenaka T, Inoue T, Kanno Y, Okada H, Meaney KR, Hill CE, Suzuki H (2008) Expression and role of connexins in the rat renal vasculature. Kidney Int 73:415-22

34. Haefliger JA, Castillo E, Waeber G et al (1997) Hypertension increases connexin43 in a tissue-specific manner. Circulation 18:1007-14

35. Haefliger JA, Krattinger N, Martin D et al (2006) Connexin43-dependent mechanism modulates renin secretion and hypertension J. Clin. Invest 116:405413

36. Gerl M, Kurt B, Kurtz A, Wagner C. (2014) Connexin 43 is not essential for the control of renin synthesis and secretion. Pflugers Arch 466:1003-9

37. Takenaka $\mathrm{T}$, Inoue $\mathrm{H}$, Okada $\mathrm{Y}$ et al (2011) Altered gap junctional communication and renal haemodynamics in Zucker fatty rat model of type 2 diabetes. Diabetologia 54:2192-2201.

38.Loeffler I, Hopfer U, Koczan D, Wolf G (2011) Type VIII collagen modulates TGF- $\beta 1$-induced proliferation of mesangial cells. J Am Soc Nephrol 22:649-63

39.Cove-Smith A, Hendry BM (2008) The regulation of mesangial cell proliferation. Nephron Exp Nephrol 108:74-9. 
40. Yao J, Zhu Y, Morioka T, Oite T, Kitamura M (2007) Pathophysiological roles of gap junction in glomerular mesangial cells. J Membr Biol 217:123-30

41. Xie X, Lan T, Chang X, et al (2013) Connexin43 mediates NF-kB signalling activation induced by high glucose in GMCs: involvement of c-Src. Cell Commun Signal 11:38

42. Yao J, Morioka T, Oite T (2000) PDGF regulates gap junction communication and connexin43 phosphorylation by PI 3-kinase in mesangial cells. Kidney Int 2000 57:1915-26

43. Morioka T, Okada S, Nameta M, et al (2013) Glomerular expression of connexin 40 and connexin 43 in rat experimental glomerulonephritis. Clin Exp Nephrol 17:191-204

44.Dalla Vestra M, Saller A, Mauer M, Fioretto P (2001) Role of mesangial expansion in the pathogenesis of diabetic nephropathy. J Nephrol 14:S51-S57

45.Zhang X, Chen X, Wu D, et al (2006) Downregulation of connexin 43 expression by high glucose induces senescence in glomerular mesangial cells. $J$ Am Soc Nephrol 17:1532-42

46.Liu L, Hu X, Cai GY, et al (2012) High glucose-induced hypertrophy of mesangial cells is reversed by connexin43 overexpression via PTEN/Akt/mTOR signaling. Nephrol Dial Transplant 27:90-100

47.Giepmans BN, Hengeveld T, Postma FR, Moolenaar WH (2001) Interaction of c-Src with gap junction protein connexin-43: role in the regulation of cell-cell communication. J Biol Chem 276:8544-8549

48.Gilleron J, Fiorini C, Carette D, et al (2008) Molecular reorganization of Cx43, $\mathrm{ZO}-1$ and Src complexes during the endocytosis of gap junction plaques in response to a non-genomic carcinogen. J Cell Sci 121:4069-4078 
49. Suzaki $Y$, Yoshizumi M, Kagami $S$ et al (2004) BMK1 is activated in glomeruli of diabetic rats and in mesangial cells by high glucose conditions. Kidney Int 65:1749-60

50. Prabhakar SS (2005) Pathogenic role of nitric oxide alterations in diabetic nephropathy. Curr Diabetes Rep 5: $449-454$

51. Awad A, Webb R, Carey R, Siragy HM (2004) Renal nitric oxide production is decreased in diabetic rats and improved by AT receptor blockade. J Hypertens 22:1571-1577

52. Prabhakar SP, Starnes J, Shi S, Lonis B, Tran R (2007) Diabetic nephropathy is associated with oxidative stress and decreased renal nitric oxide production. J Am Soc Nephrol 18: 2945-52

53. Mumtaz F, Dashwood M, Khan M, Thompson CS, Mikhailidis DP, Morgan RJ (2004) Down-regulation of nitric oxide synthase in the diabetic rabbit kidney: Potential relevance to the early pathogenesis of diabetic nephropathy. 20:1 -6

54. Yao J, Hiramatsu N, Zhu Y, Morioka T, Takeda M, Oite T, Kitamura M (2005) Nitric oxide-mediated regulation of connexin43 expression and gap junctional intercellular communication in mesangial cells. J Am Soc Nephrol 16:58-67

55.Lu D, Soleymani S, Madakshire R, Insel PA (2012) ATP released from cardiac fibroblasts via connexin hemichannels activates profibrotic P2Y2 receptors. FASEB J 26:2580-2591

56. Retamal MA, Cortés CJ, Reuss L, Bennett MVL, Sáez JC (2006) SNitrosylation and permeation through connexin 43 hemichannels in astrocytes: induction by oxidant stress and reversal by reducing agents. Proceedings of the National Academy of Sciences of the United States of America 103: 4475-4480

57.Hernández-Salinas R, Vielma AZ, Arismendi MN, Boric MP, Sáez JC, Velarde V (2013) Boldine prevents renal alterations in diabetic rats. J Diabetes Res 
58. Hillis GS, Duthie LA, Brown PA, Simpson JG, MacLeod AM, Haites NE (1997) Upregulation and co-localization of connexin 43 and cellular adhesion molecules in inflammatory renal disease. J Pathol 182:373-9

59. Hillis GS, Duthie LA, Mlynski R, et al (1997). The expression of connexin 43 in human kidney and cultured renal cells. Nephron 75:458-63

60.Yaoita E, Yao J, Yoshida Y, et al (2002) Up-regulation of connexin43 in glomerular podocytes in response to injury. Am J Pathol 161:1597-1606

61.Yan Q, Gao K, Chi Y, Li K et al (2012). NADPH oxidase-mediated upregulation of connexin43 contributes to podocyte injury. Free Radic Biol Med $15: 1286-97$

62. Sawai K, Mukoyama M, Mori K et al (2006) Redistribution of connexin43 expression in glomerular podocytes predicts poor renal prognosis in patients with type 2 diabetes and overt nephropathy. Nephrol Dial Transplant 21:2472-7

63. Hills CE, Squires PE (2011) The role of TGF- $\beta$ and epithelial-to mesenchymal transition in diabetic nephropathy. Cytokine Growth Factor Rev 22: 131-9

64. Hills CE, Squires PE (2010) TGF- $\beta 1$-induced epithelial-to-mesenchymal transition and therapeutic intervention in diabetic nephropathy. Am J Nephrol $31: 68-74$

65. Mege RM, Matsuzaki F, Gallin WJ, Goldberg JI, Cunningham BA, Edelman GM (1988) Construction of epithelioid sheets by transfection of mouse sarcoma cells with cDNAs for chicken cell adhesion molecules Proc Natl Acad Sci U S A 85:7274- 8

66. Jongen WM, Fitzgerald DJ, Asamoto M et al (1991) Regulation of connexin 43-mediated gap junctional intercellular communication by $\mathrm{Ca}^{2+}$ in mouse epidermal cells is controlled by E-cadherin. J Cell Biol 114:545-55 
67. Hills CE, Siamantouras E, Smith SW, Cockwell P, Liu KK, Squires PE (2012) TGF $\beta$ modulates cell-to-cell communication in early epithelial-to-mesenchymal transition. Diabetologia 55:812-24

68. Hills CE, Kerr MI, Wall MJ, Squires PE (2013) Visfatin reduces gap junction mediated cell-to-cell communication in proximal tubule-derived epithelial cells. Cell Physiol Biochem 32:1200-12

69. Hirschberg R (2005) Wound healing in the kidney: complex interactions in renal interstitial fibrogenesis. J Am Soc Nephrol 16:9-11

70. Liu Y (2006) Renal fibrosis: new insights into the pathogenesis and therapeutics. Kidney Int 69:213-7

71. Wright CS, Pollok S, Flint DJ, Brandner JM, Martin PE (2012) The connexin mimetic peptide Gap27 increases human dermal fibroblast migration in hyperglycemic and hyperinsulinemic conditions in vitro. J Cell Physiol 2012 227:77-87

72. Qiu C, Coutinho P, Frank S, et al (2003) Targeting connexin43 expression accelerates the rate of wound repair. Curr Biol 13:1697-1703

73. Wang CM, Lincoln J, Cook JE, Becker DL (2007) Abnormal connexin expression underlies delayed wound healing in diabetic skin. Diabetes 56:28092817

74. Mendoza-Naranjo A, Cormie P, Serrano AE et al (2012) Targeting Cx43 and $\mathrm{N}$ - cadherin, which are abnormally upregulated in venous leg ulcers, influences migration, adhesion and activation of Rho GTPases. PLoS One 7:e37374

75. Abed A, Toubas J, Kavvadas P et al (2014) Targeting connexin 43 protects against the progression of experimental chronic kidney disease in mice. Kidney Int 86:768-779

76. Sipos A, Vargas SL, Toma I, Hanner F, Willecke K, Peti-Peterdi J. (2009) 
Connexin 30 deficiency impairs renal tubular ATP release and pressure natriuresis. J Am Soc Nephrol 20:1724-32

77. Svenningsen P, Burford JL, Peti-Peterdi J (2013). ATP releasing connexin 30 hemichannels mediate flow-induced calcium signaling in the collecting duct. Front Physiol 16;4:292

78. Hills CE, Bland R, Wheelans DC, Bennett J, Ronco PM, Squires PE (2006) Glucose-evoked alterations in connexin43-mediated cell-to-cell communication in human collecting duct: a possible role in diabetic nephropathy. Am J Physiol Renal Physiol 291:F1045-F1051

79. Hills CE, Bland R, Squires PE (2012) Functional expression of TRPV4 channels in human collecting duct cells: implications for secondary hypertension in diabetic nephropathy. Exp Diabetes Res 2012:936518

80. Hills CE, Bland R, Bennett J, Ronco PM, Squires PE (2009) TGF- $\beta 1$ mediates glucose-evoked up-regulation of connexin-43 cell-to-cell communication in HCD-cells. Cell Physiol Biochem 24:177-86

81. Lee YC, Yellowley CE, Li Z, Donahue HJ, Rannels DE (1997) Expression of functional gap junctions in cultured pulmonary alveolar epithelial cells. Am J Physiol 272:L1105-L1114

Figure Legends

Fig. 1 Gap Junction assembly may be homotypic or heterotypic. Gap junction formation occurs when connexons on adjacent cells align to form either a homotypic or heterotypic structure. Whether the connexon is defined as homomeric or heteromeric is dependent on the nature of the connexin isoform present. If a gap junction comprises two identical hexameric structures, then the channel is rendered homotypic. However, if the composition of each aligning connexon is different as a consequence of multiple connexin isoforms, then the channel is defined as heterotypic 
Fig. 2 Connexins are the building blocks of both gap junctions and hemichannels. Alignment of connexons on adjacent cells (Cell 1 and Cell 2) permits electrical coupling and direct gap junction-mediated cell-cell communication of small ions and molecules (e.g. $\mathrm{Ca}^{2+}$ and cAMP, black dots). When connexons lack a binding partner on a neighbouring cell, e.g. Cell 3, undocked hemichannels provide a conduit for the efflux of these small cytosolic molecules and ions into the intercellular space immediately surrounding the cell (e.g. ATP, red dots). The limited volume of this intercellular space allows the concentration of agonists to increase ( $\uparrow$ [ATP]e) to levels capable of evoking a receptor-mediated response on adjacent cells expressing the appropriate receptor, e.g. Cell 2 expressing P2Y purinoreceptors. This local paracrine transfer of information provides an alternative route for synchronising cellular activity 